Applied Physics B manuscript No.

(will be inserted by the editor)

\title{
Photoassociation inside an optical dipole trap: absolute rate coefficients and Franck-Condon factors
}

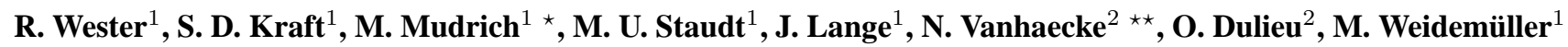 \\ 1 Physikalisches Institut, Universität Freiburg, Hermann-Herder-Straße 3, 79104 Freiburg, Germany \\ ${ }^{2}$ Laboratoire Aimé Cotton, CNRS, Bâtiment 505, Campus d’Orsay, 91405 Orsay, France
}

October 22, 2018

\begin{abstract}
We present quantitative measurements of the photoassociation of cesium molecules inside a far-detuned optical dipole trap. A model of the trap depletion dynamics is derived which allows to extract absolute photoassociation rate coefficients for the initial single-photon photoassociation step from measured trap-loss spectra. The sensitivity of this approach is demonstrated by measuring the Franck-Condon modulation of the weak photoassociation transitions into the low vibrational levels of the outer well of the $0_{g}^{-}$state that correlates to the $6 s+6 p_{3 / 2}$ asymptote. The measurements are compared to theoretical predictions. In a magneto-optical trap these transitions have previously only been observed indirectly through ionization of ground state molecules.
\end{abstract}

\section{Introduction}

Formation, manipulation and interactions of ultracold molecules today forms one of the major topics in ultracold atomic and molecular physics. Photoassociation of a cold atom pair followed by spontaneous emission to the ground state, which has started the field of ultracold molecules [?,?], and coherent magnetic field sweeps across Feshbach resonances represent the most prominent techniques to form ultracold molecules; the latter has finally lead to the successful creation of molecular Bose Einstein condensates [?,?,?]. To populate low-lying molecular states that are not accessible by the Feshbach resonance method, two-color photoassociation schemes have been investigated [?,?]. Coherent photoassociation of a Mott insulator phase with exactly two atoms per lattice site into a molecular phase has combined the two approaches employing the scheme of a laser-driven Feshbach resonance [?]. The formation of heteronuclear cold molecules composed of two

\footnotetext{
* Present address: Laboratoire Aimé Cotton, CNRS, Bâtiment 505, Campus d'Orsay, 91405 Orsay, France

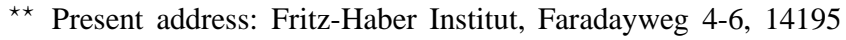
Berlin, Germany
}

different alkali atoms has only recently been successfully achieved by photoassociation in a double magneto-optical trap [?,?].

We have investigated photoassociation inside a far-detuned optical dipole trap, because this type of trap offers the possibility to catch associated molecules that decay into bound levels of the ground electronic state inside the same optical dipole trap and store or manipulate them for long periods of time. This approach will therefore facilitate collision and chemical reaction experiments with state-selected ultracold molecules and may culminate in coherent ultracold chemistry [?]. While most photoassociation experiments so far have employed magneto-optical traps, the first demonstration of the photoassociation of ultracold atoms employed an optical dipole trap [?]. The higher densities and the suppression of near resonant light scattering in optical dipole traps allow for high photoassociation rates and long photoassociation times. As a consequence, a strong loss of trapped atoms due to photoassociation is achieved. Furthermore, the initial atomic quantum state is not determined by the trap, in contrast to magneto-optical or magnetic traps, but can be controlled independently.

In this work we present photoassociation trap loss spectra of ultracold cesium inside a quasi-static optical dipole trap formed by a $\mathrm{CO}_{2}$ laser. We focus on the $0_{g}^{-}\left(6 s+6 p_{3 / 2}\left({ }^{2} P_{3 / 2}\right)\right)$ double-well state of $\mathrm{Cs}_{2}$ (hereafter referred to as $0_{g}^{-}\left(P_{3 / 2}\right)$ ) which is extensively described in Ref. [?]. Fig. 1 schematically shows this molecular potential curve together with the curves of other relevant electronic states that correlate to the $6 s+6 p_{3 / 2}$ asymptote. The $0_{g}^{-}\left(P_{3 / 2}\right)$ state is particularly interesting, because single-photon photoassociation allows to populate vibrational levels in its outer well that have a high probability to decay into bound levels of the electronic ground states. This is due to the barrier between the inner and outer well of this potential which leads to large Franck-Condon factors for the radiative decay [?]. Also tunneling into the inner part of the potential has been observed [?]. The highly sensitive detection of ground state molecules by multiphoton ionization was used in a sequence of experiments by P. Pillet et al. to study the photoassociation of $\mathrm{Cs}_{2}[?, ?, ?]$. 
Modeling the trap depletion dynamics as a function of photoassociation time allowed us to extract precise absolute photoassociation rate coefficients, i. e. with an accuracy of better than a factor of 2 [?]. We have observed photoassociation resonances down to the lowest vibrational levels of the $0_{g}^{-}\left(P_{3 / 2}\right)$ outer well. These resonances have very small rate coefficients and could only be detected in previous measurements, because they decay into bound molecules and are therefore observed by multiphoton ionization. Our measurement yield the same quality as the ion spectra of Ref. [?] without introducing the radiative transition to bound molecules and the ionization efficiency into the signal strength. This allows us to extract the Franck-Condon modulation of the initial photoassociation step for all vibrational levels down to the $v=0$ level of the $0_{g}^{-}\left(P_{3 / 2}\right)$ external well.

\section{Experimental setup}

The photoassociation experiments have been performed with an ensemble of cesium atoms trapped in an optical dipole trap that is formed by the focus of a $\mathrm{CO}_{2}$ laser. As described in detail in Refs. [?,?], the optical dipole trap is loaded from a cesium magneto-optical trap which is superimposed in the focus of the $\mathrm{CO}_{2}$ laser (see Fig. 2). The MOT is operated in five-beam configuration and loads from a Zeeman slowed beam up to $10^{8}$ particles at a density of $10^{9} \mathrm{~cm}^{-3}$ as inferred from absorption imaging.

After turning off the magnetic field of the MOT a brief molasses cooling phase transfers about $5 \times 10^{5}$ atoms at a density of $5 \times 10^{11} \mathrm{~cm}^{-3}$ and a temperature of $40 \mu \mathrm{K}$ into the optical dipole trap [?,?]. The repumping beam is extinguished several milliseconds before the main MOT beam, which pumps all $\mathrm{Cs}$ atoms into the $F=3$ ground state. For the cold cesium atoms the $\mathrm{CO}_{2}$ laser focus with its potential depth of $0.8 \mathrm{mK}$ represents a harmonic trap with axial and radial trap frequencies of $\omega_{a x}=12.8 \mathrm{~Hz}$ and $\omega_{\text {rad }}=625 \mathrm{~Hz}$ respectively. In thermal equilibrium the density distribution of the atoms in the trap is given by a cylindrically symmetric Gaussian distribution with standard deviations of $600 \mu \mathrm{m}$ and $13 \mu \mathrm{m}$ in the axial and radial directions, respectively. The lifetime of the atoms in the dipole trap is of the order of $100 \mathrm{~s}$, due to collisions with residual gas atoms.

The photoassociation light is provided by a widely tunable Titanium:Sapphire laser (Coherent MBR 110) system, which delivers a typical output power of $200 \mathrm{~mW}$ with a line width of about $100 \mathrm{kHz}$, after passing through an optical isolator and a single-mode optical fibre. Relative frequency changes are monitored by measuring the transmission signal of a fraction of the Ti:Sapphire laser beam through a confocal cavity with a free spectral range of $1000 \mathrm{MHz}$. In order to decrease the frequency spacing of the transmission peaks, two sidebands are modulated onto the cavity path of the Ti:Sapphire beam at $\pm 200 \mathrm{MHz}$ using a double-pass AOM setup. The length of the cavity is stabilized by locking one of the cavity mirrors with a piezo actuator to the fringe of the transmission signal of a superimposed cesium-spectroscopy stabilized diode laser. This provides a relative frequency accuracy of about $5 \mathrm{MHz}$. The absolute laser frequency is measured with a commercial wavemeter (Burleigh WA 1000) with an accuracy of $500 \mathrm{MHz}$. The photoassociation laser is passed through the trapped cesium cloud in the focus of the $\mathrm{CO}_{2}$ laser at an angle of $\theta=22.5^{\circ}$ with respect to the $\mathrm{CO}_{2}$ laser beam. The width of the Ti:Sapphire beam at the trap center amounts to $150 \mu \mathrm{m}$. The intensity of the photoassociation beam amounted to typically $50 \mathrm{~W} / \mathrm{cm}^{2}$ and was increased for the weakest vibrational transitions up to $300 \mathrm{~W} / \mathrm{cm}^{2}$.

Once the cesium atoms are loaded into the dipole trap, the shutter of the photoassociation laser is opened and the atom cloud is illuminated for up to $1000 \mathrm{~ms}$. For some data sets a short molasses cooling pulse is applied after half of the photoassociation time to lower the temperature and thereby increase the photoassociation rate. Finally the $\mathrm{CO}_{2}$ laser light is extinguished and all remaining cesium atoms are recaptured into the magneto-optical trap. The number of recaptured cesium atoms is obtained from the fluorescence signal of the MOT with a relative accuracy of better than $5 \%$. The fluorescence signal is calibrated to the absolute atom number using absorption images, which leads to an absolute accuracy of the atom number of $30 \%$. Due to the very long storage time, the photoassociation-laser induced atom loss signal is a precise measure for the formation of cold molecules through photoassociation. To measure photoassociation spectra the frequency of the Ti:Sapphire laser is scanned and the loading, photoassociation and recapture cycle is repeated. The frequency range scanned during one cycle is $3 \mathrm{MHz}$.

A wide photoassociation spectrum, composed of several individual scans, is shown in the lower trace of Fig. 3 It is compared to a photoassociation scan obtained directly in the magneto-optical trap using a lock-in scheme to measure the fluorescence reduction that occurs when a photoassociation resonance adds a loss channel to the MOT (upper trace). The spectra show vibrational progressions in the $0_{g}^{-}, 0_{u}^{+}$and $1_{g}$ molecular states that correlate to the $6 s+6 p_{3 / 2}$ asymptote. Note the exquisite stability of trap loading (overall fluctuations in particle number $<5 \%$ ). This comparison shows a significant improvement of the signal-to-noise ratio for the optical dipole trap measurement. Furthermore, the Cs hyperfine state in the MOT is $F=4$, whereas in the dipole trap it was chosen to be the absolute ground state, $F=3$. This is reflected in the different amplitudes of the resonances in the MOT compared to the dipole trap.

\section{Photoassociation results}

\subsection{Photoassociation into the $0_{g}^{-}\left(P_{3 / 2}\right)$ outer well}

By measuring photoassociation induced trap loss from the optical dipole trap, the vibrational progression of the $0_{g}^{-}\left(P_{3 / 2}\right)$ outer well of $\mathrm{Cs}_{2}$ (see Fig. 1 has been observed. Selected photoassociation (PA) spectra are shown in Fig. 4 for the vibrational levels $v=2,6$ and 10 . It can be seen that the number of recaptured atoms drops significantly when the PA laser is tuned to a resonance with a rovibrational state, leading to 
a strong spectroscopic signature. Outside of a resonance essentially $100 \%$ of the atoms transferred to the dipole trap are recaptured. The noise on the baseline corresponds to the shotto-shot fluctuations in the number of atoms transferred to the dipole trap.

In the $0_{g}^{-}$state narrow resonances are observed, showing a rotational progression for each vibrational level. Vibrational quantum numbers are assigned to each spectrum based on the accurate spectroscopic investigation of Ref. [?]. From the rotational progression for each vibrational level rotational constants are extracted, which are found to be in good agreement with previous studies (see table 1 ).

It is interesting to note that we observe rigid rotor states of the cesium molecule up to $J=4$. For pure $s$-wave scattering angular momentum conservation predicts a maximum rotational excitation of $J=2$ for optical dipole excitation from the triplet ground state. This is indeed the strongest rotational line in all the photoassociation spectra. Larger rotational states can be rationalized due to additional angular momentum contributions, such as from orbital angular momentum due to $p$-wave or $d$-wave scattering or from the total nuclear spin of the atom pair. Actually, the height of the $p$-wave centrifugal barrier is estimated to be $35 \mu \mathrm{K}$, assuming that the initial collisional channel proceeds via the $a^{3} \Sigma_{u}^{+}(6 s+$ $6 s)$ state only. Given the initial temperature of the cesium atoms of $40 \mu \mathrm{K}$, this leads us to attribute the $J=3$ resonances to $p$-wave collisions. During the photoassociation time in the trap the temperature increases to about $60 \mu \mathrm{K}$, as described in detail below. The origin of the weak $J=$ 4 component may therefore be a $d$-wave collision (barrier height about $200 \mu \mathrm{K}$ ) of two fast atoms in the thermal distributions that either tunnel through or surmount the barrier, even though both the $p$ - and $d$-wave barriers are found too weak to support a shape resonance that could increase the tunneling probability. Light-induced mechanisms such as "flux enhancement" which are important in magneto-optical traps [?] do not play a role in a far-detuned optical dipole trap.

\subsection{Model of the trap loss dynamics}

Owing to the controlled conditions that prevail inside the optical dipole trap it is feasible to convert the trap loss signal into an absolute photoassociation rate coefficient. For high photoassociation laser intensities or long interaction times the photoassociation process can easily deplete more than $50 \%$ of the trapped atoms. Therefore a model is needed to describe the depletion of the trapped atoms. This model assumes photoassociation from an atomic cloud at thermal equilibrium and takes into account the time dependence of both the atomic density and the temperature inside the optical dipole trap. The basis of the depletion model is the rate equation for trap loss

$$
\frac{d N}{d t}=-2 \int G(\mathbf{x}, T) n^{2}(\mathbf{x}, T) d^{3} x
$$

which describes the loss of two atoms per collision event and depends on the photoassociation rate coefficient $G(\mathbf{x}, T)$ and the pair density $n^{2}(\mathrm{x}, T)$. The latter is given by the square of the cylindrically symmetric Gaussian density distribution of the thermal trapped atom cloud. The rate coefficient $G(\mathbf{x}, T)$ needs special attention, because it depends on the photoassociation laser intensity and therefore becomes position dependent if the PA laser intensity varies spatially. In the experiment the width of the PA laser of $w=150 \mu \mathrm{m}$ is much larger than the radial extension of the atomic cloud $(13 \mu \mathrm{m})$, but smaller than the axial extension $(800 \mu \mathrm{m})$. The intensity therefore changes only along the axial direction, denoted $z$, and has a Gaussian profile. In the low-intensity limit $(G \propto$ intensity) one obtains

$$
G(\mathbf{x}, T)=G_{0}(T) e^{-2 \sin ^{2} \theta z^{2} / w^{2}} .
$$

Note that the width of the distribution is given by the PA laser width and the intersection angle of the PA laser with the trap axis $\theta$. The peak rate coefficient $G_{0}(T)$ corresponds to the peak intensity $2 P /\left(\pi w^{2}\right)$ of the photoassociation laser with power $P$.

Integration of Eq. (1) yields a differential equation for the particle number $N$,

$$
\frac{d N}{d t}=-2 G_{0}(T) \frac{N^{2}}{V(T)},
$$

where the effective volume $V(T)$ depends on the PA laser and $\mathrm{CO}_{2}$ trap parameters and is given by

$$
V(T)=\left(\frac{2 \pi k_{B} T}{m_{C s} \omega_{r a d} \omega_{a x}}\right)^{2} \frac{\sqrt{8} \sin \theta}{\sqrt{\pi} w}=V_{0} T^{2} .
$$

The connection between particle number and temperature is taken from a thermodynamic model of the trap depletion which was also successfully employed to study hyperfinechanging Cs-Cs and Li-Cs collisions [?]. This model assumes that each colliding atom pair upon leaving the trap removes a total energy of $q 6 k_{B} T$ where the parameter $q$ is obtained from a fit to the experimental data yielding $0.5 \pm 0.05$. As a consequence of this relation, temperature and particle number are linked by the scaling law

$$
\frac{T}{T_{0}}={\frac{N}{N_{0}}}^{q-1}
$$

Given the temperature dependence of the rate coefficient at low temperatures $G_{0}(T) \propto T^{-\frac{1}{2}}$, Eq. (3) is integrated analytically yielding the number of trapped atoms and the temperature as a function of the storage time and the photoassociation rate coefficient. This is inverted to give the rate coefficient for the initial temperature $T_{0}$ as a function of the remaining atoms $N\left(t_{P A}\right)$ after a photoassociation time $t_{P A}$,

$$
G_{0}\left(T_{0}\right)=\frac{V_{0} T_{0}^{2}}{(9 / 2) N_{0} t_{P A}}\left(\left(N\left(t_{P A}\right) / N_{0}\right)^{-9 / 4}-1\right) .
$$

The cooling pulse that has been applied for some data sets after $t_{P A} / 2$ was observed to reduce temperature and particle number of the trapped atoms by a factor of $\alpha \sim 0.75$. It is accounted for by a piece-wise solution of the differential 
R. Wester et al.

equation (3) and leads for these data sets to a correction factor of $1 /\left(1+\alpha^{3 / 2}\right) \sim 0.4$ to the rate coefficient $G_{0}$.

The trap depletion model was verified experimentally for a single photoassociation resonance by measuring the atom number, the atomic density and the temperature as a function of photoassociation time using absorption imaging. The result in Fig. 5 shows the measured decrease of the number of cesium atoms. As can be seen, the temperature of the atoms is increasing from 40 to about $80 \mu \mathrm{K}$ during $10 \mathrm{~s}$ of interaction time with the photoassociation laser, which is due to the coldest atoms associating most favorably. Within the photoassociation time of $1 \mathrm{~s}$ that is employed to measure photoassociation spectra, the temperature increases to about $60 \mu \mathrm{K}$. This effect is included in the model through Eq. (5). One can see that the agreement to our trap depletion model is satisfactory, despite its simplicity.

Using this model, specifically Eq. 6 absolute photoassociation rate coefficients are calculated for the resonances of the $v=2$ spectrum in Fig. 4 and values between 5 . $10^{-12} \mathrm{~cm}^{3} / \mathrm{s}$ for $J=0$ and $3 \cdot 10^{-11} \mathrm{~cm}^{3} / \mathrm{s}$ for $J=2$ are obtained. The PA laser intensity amounted to $90 \mathrm{~W} / \mathrm{cm}^{2}$. The accuracy of the obtained rate coefficients is estimated to be about $40 \%$, which is mainly due to the accuracy of the absolute particle number as obtained from absorption imaging (estimated to be $30 \%$ ) and the accuracy of the cesium temperature (estimated to be $10 \%$ ).

In Ref. [?] absolute photoassociation rate coefficients were measured in a magneto-optical trap and compared to theoretical calculations. The measurements were carried out for several PA resonances in the $0_{u}^{+}$and $0_{g}^{-}$states at small detunings and rate coefficients of between $1 \cdot 10^{-11} \mathrm{~cm}^{3} / \mathrm{s}$ and $5 \cdot 10^{-11} \mathrm{~cm}^{3} / \mathrm{s}$ were measured. In the same paper theoretical rate coefficients are calculated using a perturbative model in good agreement with the measured rates. For photoassociation into the $0_{g}^{-}$state at large detunings, corresponding to the low vibrational levels that are studied here, the model in Ref. [?] predicts rate coefficients of a few times $10^{-12} \mathrm{~cm}^{3} / \mathrm{s}$ for a laser intensity of $55 \mathrm{~W} / \mathrm{cm}^{2}$. Our measured rate coefficients are somewhat larger than this range. This is partly accounted for by the higher laser intensity by a factor of 1.5 and the lower temperature in our experiment of almost a factor of 4 which together predicts higher rate coefficient by about a factor of three or values of around $10^{-11} \mathrm{~cm}^{3} / \mathrm{s}$ [?]. This is in reasonable agreement with the measured values. An even better test of the quality of the absolute rate coefficient measurements in the dipole trap has been carried out in our group by studying saturation of the photoassociation rate coefficient at the unitarity limit [?]. These measurements agree within the estimated $40 \%$ accuracy with theoretical prediction for the unitarity limited photoassociation rate coefficient.

\subsection{Franck-Condon modulation in the $0_{g}^{-}\left(P_{3 / 2}\right)$ outer well}

The photoassociation efficiency depends crucially on the FranckCondon (FC) factor $\langle\psi(v) \mid \phi\rangle$ involving the continuum wavefunction $|\phi\rangle$ of the initial collisional state and the molecular wavefunction $|\psi(v)\rangle$ of the PA level in the excited molecular state. The brackets hold for the integration over the interatomic coordinate $R$. In this approach the generally $R$ dependent dipole transition moment is approximated to be constant for the interatomic distances at which photoassociation occurs. The FC factor is known to exhibit strong variations with the vibrational quantum number $v$ of the excited state $[?, ?, ?]$. Indeed, the PA process involves an excited potential curve and an initial potential curve varying as $R^{-3}$ and $R^{-6}$ respectively at large distances. For small detunings of the PA laser, the overlap integral is mainly determined by the value of the initial continuum wavefunction at the external turning point of the excited potential. The intensity pattern of the PA spectrum then reflects the oscillations of the continuum wavefuntion. At larger detunings, radial wave functions in both channels may locally oscillate with similar frequency, inducing abrupt changes in the overlap integral.

In this work we present measurements of the Franck-Condon modulation directly through the analysis of trap loss spectra for the lowest vibrational levels in the $0_{g}^{-}\left(P_{3 / 2}\right)$ external well, in contrast to previous measurements [?] which detected ground state molecules by photoionization and therefore measured the FC modulation multiplied by the radiative decay probability and the ionization efficiency. Furthermore, in the present experiment the $F=3$ initial state is chosen for the cesium atoms, in contrast to the measurements with $F=4$ atoms in a magneto-optical trap.

To connect the photoassociation rate coefficient with the Franck-Condon factor we employ the theoretical description by Bohn and Julienne [?], which is, for low photoassociation intensities, equivalent to the treatment proposed in several papers $[?, ?, ?]$. It yields a scattering probability

$$
p_{s c}=\frac{G}{G_{\text {unitarity }}}=\frac{\Gamma}{\gamma / 4} .
$$

This relation holds if the PA rate coefficient $G$ is small compared to the unitarity limited maximum rate PA coefficient $G_{\text {unitarity }} \propto T^{-1 / 2}$. The scattering probability $p_{s c}$ thus depends on the coupling constant $\Gamma$ and the natural line width $\gamma$ of the electronic transition. The coupling constant $\Gamma$ is given by

$$
\Gamma=2 \pi\left(V_{e g}\right)^{2}\left|\left\langle\psi(v) \mid \phi\left(E_{\mathrm{kin}} \rightarrow 0\right)\right\rangle\right|^{2},
$$

where $\left(V_{e q}\right)^{2}$ is the Rabi frequency squared of the electronic transition, which is proportional to the laser intensity.

For each $J=2$ rotational state of the vibrational levels $v=0$ to 23 we have measured the photoassociation rate coefficient using the trap depletion model described above. In the course of this work we investigated if more vibrational levels could be found below the $v=0$ level, which would cause an incorrect assignment of the vibrational progression. However, no additional vibrational levels were found for larger detunings than the $v=0$ level. For each observed vibrational level the Franck-Condon (FC) factor is calculated using Eqs. (7) and (8). The measurements for $v \leq 10$ were carried out with an intermediate cooling pulse and were corrected accordingly (see section 3.2). The accuracy of the individual FC factors is 
estimated to be $60 \%$, mainly given by the accuracy of the rate coefficient (see above) and the PA laser intensity. To remove the energy-normalization of the continuum wavefunction $\phi$ all FC factors have been normalized to the maximum FC factor, $v=4$, which now amounts to 1 . The result is shown in the upper panel of Fig. 6. The measured FC factors show a clear step modulation starting with a very small value for $v=0$ and 1 and increasing by a factor of 100 from $v=1$ to 2 . For higher vibrational levels a much weaker modulation is observed with a local maximum near $v=4$ and a local minimum around $v=18$.

Theoretical modeling of the Franck-Condon factor modulation has been carried out for comparison with the experimental results. This requires the computation of the $|\psi(v)\rangle$ and $|\phi\rangle$ radial wave functions. The vibrational wavefunctions of the $0_{g}^{-}\left(P_{3 / 2}\right)$ external well are easily obtained from a standard Numerov integration using the Rydberg-Klein-Rees (RKR) potential of Ref. [?]. In principle, the main task is the integration of the continuum wave function. Only when the atoms are prepared in the fully stretched states $F=4, M_{F}=4$, the collisional entrance channel is fully correlated to a single state, i.e. the lowest $a^{3} \Sigma_{u}^{+}$state: it is described accurately enough by an available theoretical potential curve, connected at large distances to an accurate $\sum C_{n} / R^{n}$ asymptotic expansion (see for instance Refs. [?,?]), and adapted to reproduce the measured cesium triplet scattering length [?]. This type of calculations has been performed in Ref. [?] and is referred to in the following as the single-component calculation.

In the present experiment the atoms are not polarized, so that many channels with different internal hyperfine quantum numbers contribute to the process. Moreover, each collisional channel is described by a radial wavefunction which results from the coupling between components of both $a^{3} \Sigma_{u}^{+}$ and $X^{1} \Sigma_{g}^{+}$electronic states, interacting through the hyperfine hamiltonian. Indeed the vibrational motion of the excited levels presented here proceeds over the $15-30 a_{0}$ range, which is the recoupling range for the hyperfine interactions in cesium. The complexity of this coupled channel problem has lead us to perform the calculation for two colliding atoms occupying the $F=3, M_{F}=3$ state. The total continuum wavefunction is then a three-component molecular wavefunction characterized by the projection $M=6$ of the total angular momentum. With this multi-component wavefunction the Franck-Condon modulation is calculated.

In the lower panel of Fig. 6 the Franck-Condon factors are shown that are calculated using the multi-component wave function (full squares) together with the result for the singlecomponent wave function (open squares). Again all values are normalized to the maximum FC factor. Both calculations clearly show a strong increase for small vibrational levels similar to the observation in the experiment. For the multicomponent calculation the steepest increase is observed between $v=2$ and 3 in contrast to the experimentally found step between $v=1$ and 2, which is also found in the singlecomponent calculation. For larger vibrational levels the FC factors of the multi-component calculation exhibit a somewhat larger amplitude variation than the measured data, but the overall FC factors are fairly constant. Even thought the atoms in the experiment are not polarized in contrast to the calculation, a similar trend is observed in the experimental data. A local minimum near $v=18$ is found in the multicomponent calculation, similar to the measurement, but other local minima near $v=7$ and 11 are not found in the measurement. The single-component calculation shows much deeper minima for $v=14$ and 21, which are not present in the more accurate multi-component calculation. Thus one may speculate that also the minima in the multi-component FC calculation may become filled in by angular momentum components that are not included in the calculation but certainly present in the experiment. To resolve this a full FC calculation or a measurement with polarized atoms would be necessary.

\section{Conclusions}

As it was shown in Ref. [?], photoassociation experiments benefit significantly from the utilization of a conservative optical trap, due to the high pair density and the long interaction times. Furthermore the internal hyperfine state of the trapped atoms is well defined. In this work we present a thorough quantitative analysis of the photoassociation in a far-detuned optical dipole trap. Using a thermodynamic trap depletion model absolute photoassociation rate coefficients were obtained with an accuracy of about $40 \%$.

The high sensitivity of photoassociation in a dipole trap was demonstrated by studying photoassociation resonances into the lowest vibrational levels of the outer well of the $0_{g}^{-}\left(P_{3 / 2}\right)$ state through trap loss. From these measurements the FranckCondon factors for the initial single-photon excitation step of the photoassociation process were extracted for the lowest 24 vibrational levels of the $0_{g}^{-}\left(P_{3 / 2}\right)$ outer well and compared to a theoretical calculation. Previously, these transitions could only be observed by multiphoton ionization of ground state molecules. No further photoassociation resonances at larger detuning than $v=0$ were found, confirming the assignment in Ref. [?].

In the current experiment ground state $\mathrm{Cs}_{2}$ molecules are presumably already produced and trapped, but not observed due to the lack of an appropriate detection system. In future experiments multiphoton ionization will be used to detect and study these trapped molecules.

\section{Acknowledgments}

The experiments were performed while the group was still at the Max-Planck-Institut für Kernphysik in Heidelberg and we wish to thank the institute, in particular D. Schwalm, for generous support. This work is supported by the Deutsche Forschungsgemeinschaft in the Schwerpunktprogramm 1116 ,Interactions in Ultracold Atomic and Molecular Gases" under WE-2661/1-2. We also acknowledge support by the EU research training network ,Cold molecules” (COMOL), under the contract number HPRN-2002-00290. 


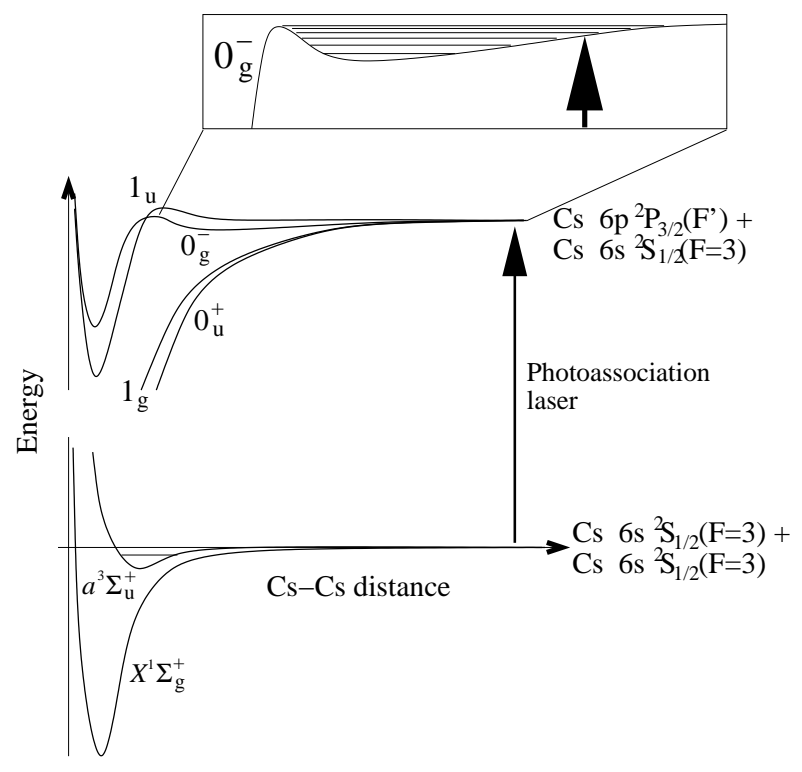

Fig. 1 Schematic overview of the relevant molecular potential curves for $\mathrm{Cs}_{2}$ photoassociation into the $0_{g}^{-}$outer well.

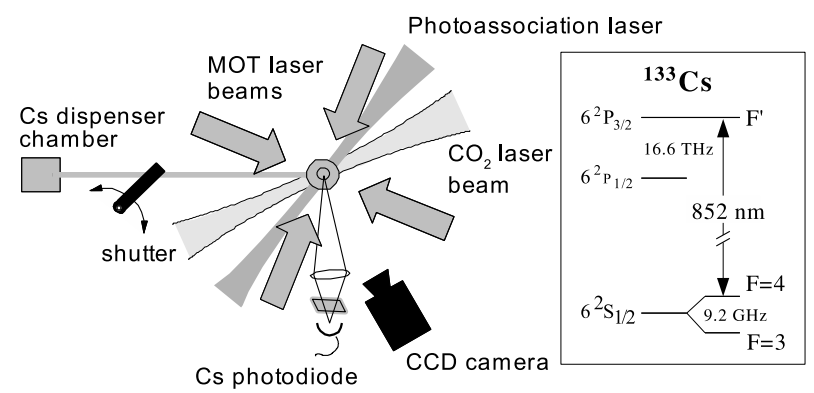

Fig. 2 Experimental setup consisting of a cesium magneto-optical trap overlapped with a $\mathrm{CO}_{2}$ optical dipole trap and a photoassociation laser.

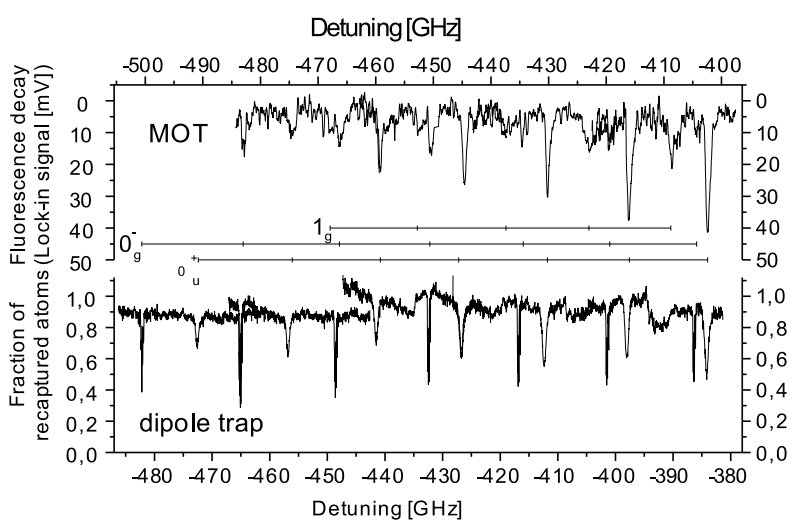

Fig. 3 Comparison of a wide-scan photoassociation spectrum measured in the far-detuned optical dipole trap (lower trace) with the same spectrum obtained in the magneto-optical trap (MOT) shown in the upper trace.

\begin{tabular}{|c|c|c|c|c|}
\hline $\mathrm{v}$ & $\delta\left[^{\mathrm{GHz}}\right]^{a}$ & $\delta[\mathrm{GHz}]^{b}$ & $B\left[^{\mathrm{MHz}}\right]^{a}$ & $B[\mathrm{MHz}]^{b}$ \\
\hline 2 & -2207.6 & $-2207.6(0.5)$ & 48.9 & $49.3(1.0)$ \\
6 & -2008.2 & $-2008.1(0.5)$ & 46.8 & $45.6(1.0)$ \\
10 & -1821.6 & $-1821.6(0.5)$ & 45.0 & $44.6(1.0)$ \\
\hline
\end{tabular}

Table 1 Detuning $\delta$ of the $J=0$ level relative to the $6 s^{2} S_{1 / 2}(F=$ $4) \rightarrow 6 p^{2} P_{3 / 2}\left(F^{\prime}=5\right)$ asymptote and rotational constants $B$ for selected vibrational states of the $0_{g}^{-}\left(P_{3 / 2}\right)\left({ }^{a}\right.$ : Ref. [?]; ${ }^{b}$ : this work, estimated accuracies are denoted in brackets).

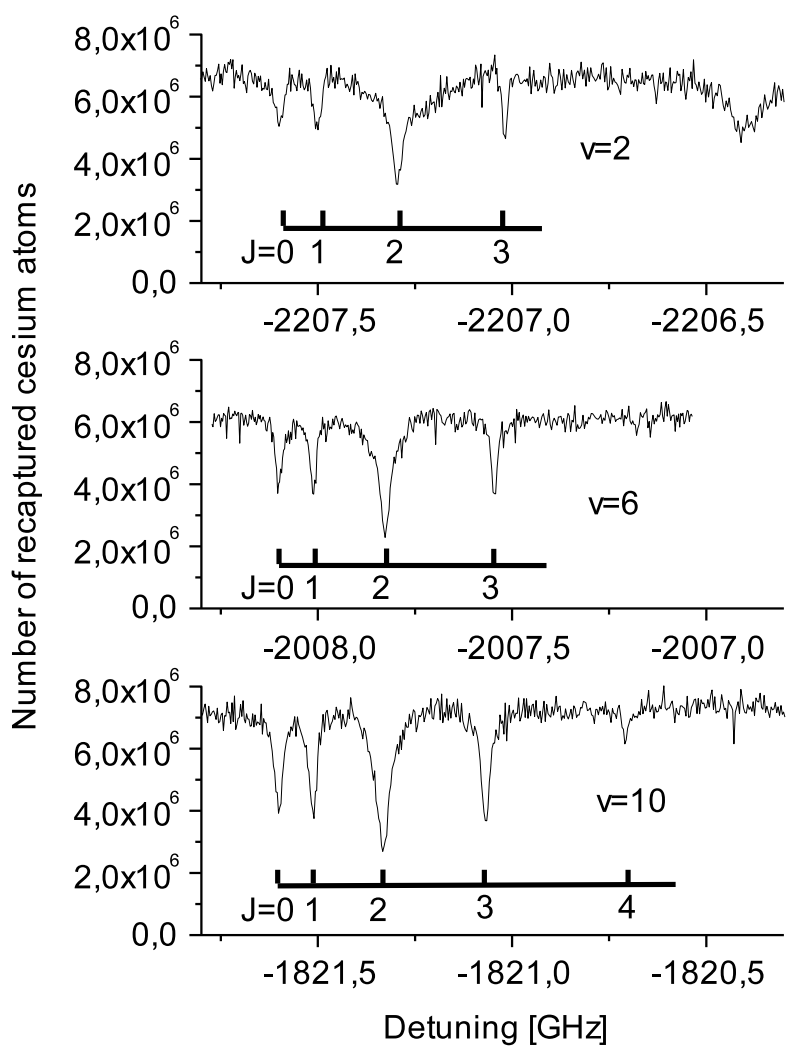

Fig. 4 Photoassociation trap loss spectra as a function of the photoassociation laser detuning relative to the $6 s^{2} S_{1 / 2}(F=4) \rightarrow 6 p$ ${ }^{2} P_{3 / 2}\left(F^{\prime}=5\right)$ asymptote for $\mathrm{v}=2,6$ and 10 in the $0_{g}^{-}$outer well. Rotational progressions are observed for each vibrational level up to $\mathrm{J}=3$. For $v=10$ also a weak $\mathrm{J}=4$ transition is observed. The resonance at $-2208 \mathrm{GHz}$ detuning in the upper panel is attributed to another electronic state. 


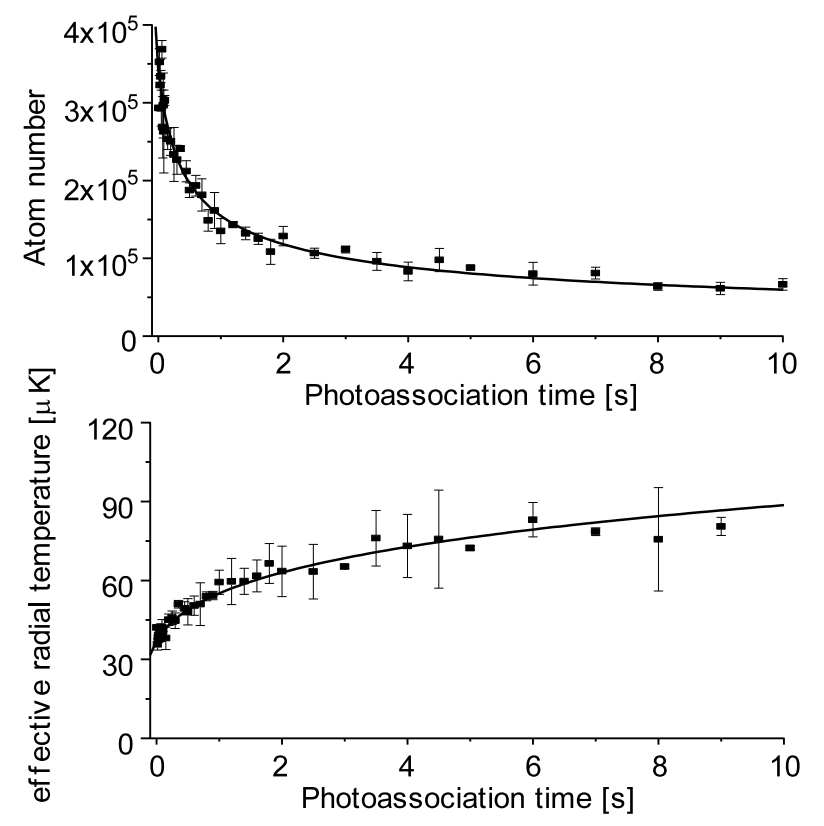

Fig. 5 Upper panel: number of atoms remaining in the trap as a function of the photoassociation laser interaction time; the solid line is a fit to the trap depletion model from which the absolute photoassociation rate coefficient is obtained. Lower panel: translational temperature of the remaining atoms as a function of photoassociation time, measured by absorption imaging of the radial expansion after release of the atoms from the $\mathrm{CO}_{2}$ trap; the solid line is the fit of the trap depletion model with the initial temperature as the only free parameter. Data are shown for the $v=20, J=2$ photoassociation resonance in the $0_{g}^{-}$outer well.

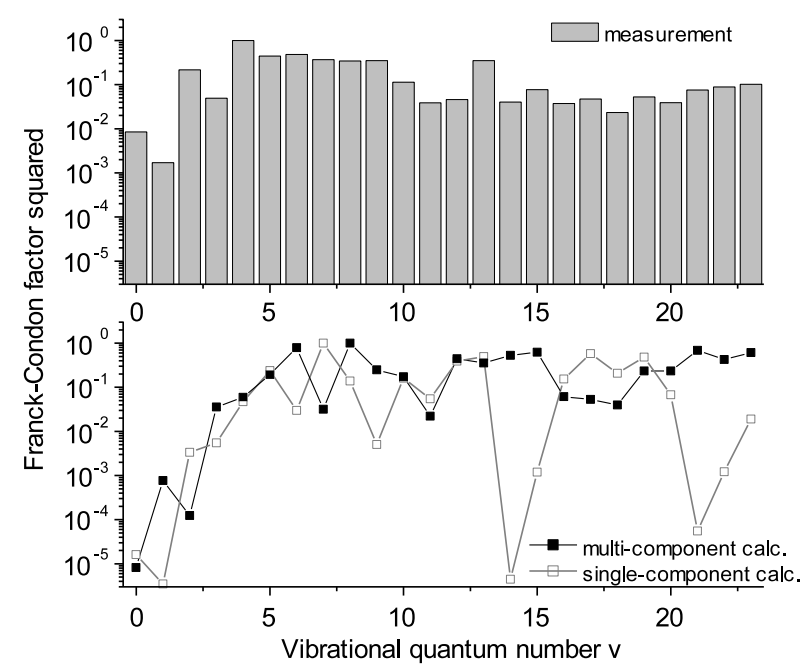

Fig. 6 Experimental data (upper panel) and theoretical results (lower panel) for the square of the Franck-Condon factor as a function of the vibrational level for photoassociation resonances into $J=2$ states in the $0_{g}^{-}$outer well. Each curve is normalized to the maximum Franck-Condon factor. 